\section{Cell type specificity of PI3K signaling in Pdk1- and Pten-deficient brains}

\author{
Nader Chalhoub, ${ }^{1}$ Guo Zhu, ${ }^{1,2}$ Xiaoyan Zhu, ${ }^{1}$ \\ and Suzanne J. Baker ${ }^{1,2,3}$ \\ ${ }^{1}$ Department of Developmental Neurobiology, St. Jude \\ Children's Research Hospital, Memphis, Tennessee 38105, USA; \\ ${ }^{2}$ Integrated Program in Biomedical Sciences, The University of \\ Tennessee Health Science Center, Memphis, Tennessee 38105, \\ USA
}

Loss of PTEN causes unregulated activation of downstream components of phosphatidylinositol 3-kinase (PI3K) signaling, including PDK1, and disrupts normal nervous system development and homeostasis. We tested the contribution of Pdk1 to the abnormalities induced by Pten deletion in the brain. Conditional deletion of Pdk1 caused microcephaly. Combined deletion of Pdk1 and Pten rescued hypertrophy, but not migration defects of Pten-deficient neurons. Pdk1 inactivation induced strikingly different effects on the regulation of phosphorylated Akt in glia versus neurons. Our results show Pdk1dependent and Pdk1-independent abnormalities in Ptendeficient brains, and demonstrate cell type specific differences in feedback regulation of the ubiquitous PI3K pathway.

Supplemental material is available at http://www.genesdev.org.

Received March 11, 2009; revised version accepted June 1, 2009.

The phosphatidylinositol 3-kinase (PI3K) signaling pathway is activated by extracellular signals and regulates crucial biological processes including metabolism, survival, proliferation, growth, and migration. Activated PI3K phosphorylates phosphatidylinositol 4,5-bisphosphate (PIP2) to generate phosphatidylinositol 3,4,5 trisphosphate (PIP3), and the lipid phosphatase PTEN reverses this reaction, thus negatively regulating the pathway (Engelman et al. 2006).

PTEN is an important tumor suppressor in sporadic brain, endometrium, and prostate cancers. In addition, inherited mutations in PTEN cause cancer predisposition, macrocephaly, and hamartomas in multiple tissues (Chalhoub and Baker 2009). In mice, germline deletion of Pten causes embryonic lethality, while heterozygous mice are tumor-prone (Di Cristofano et al. 1998; Suzuki et al. 1998; Podsypanina et al. 1999).

PDK1 is required to phosphorylate and activate AKT, a key downstream effector of PI3K signaling, in response to elevated PIP3. PDK1 also phosphorylates additional downstream substrates including S6K and SGK in a PIP3-

[Keywords: PI3K; Pdk1; Pten; brain; feedback; migration] ${ }^{3}$ Corresponding author.

E-MAIL Suzanne.Baker@StJude.org; FAX (901) 595-2270.

Article is online at http://www.genesdev.org/cgi/doi/10.1101/gad.1799609. independent manner (Rintelen et al. 2001; Mora et al. 2004). Pdk1 is indispensible in development, as germline deletion causes embryonic lethality (Lawlor et al. 2002). The PI3K pathway is evolutionarily conserved. PTEN and PDK1 are epistatic in Drosophila, in which $d P D K 1$ deficiency rescued the lethality of $d P T E N$-deficient flies (Rintelen et al. 2001). Similarly, Pdk1 is an important downstream mediator of Pten-deficient cancer in mice, where Pdk1 haploinsufficiency delayed the onset of multiple tumor types in Pten ${ }^{+-}$mice (Bayascas et al. 2005). In addition to tumorigenic phenotypes, loss of Pten or Pdk1, which activates or abrogates PI3K signaling, respectively, can influence cell size and/or cell number depending on the context of tissue type and developmental stage analyzed (Williams et al. 2000; Lawlor et al. 2002; Mora et al. 2003, 2005; Hashimoto et al. 2006; Haga et al. 2008; Chalhoub and Baker 2009|.

Conditional deletion of Pten in the mouse CNS has elucidated multiple roles in brain development and maintenance, causing increased cell proliferation and size, and neuronal migration defects (Groszer et al. 2001; Li et al. 2002; Marino et al. 2002; Fraser et al. 2004; Yue et al. 2005). Pten deletion in neural progenitors resulted in enhanced proliferation and self-renewal in vitro (Groszer et al. 2001). However, Pten deletion in post-mitotic neurons caused hypertrophy without effects on proliferation. In these models, pronounced macrocephaly was attributable to a cell-autonomous effect of Pten loss in neurons (Backman et al. 2001; Kwon et al. 2001), or a wider effect of Pten loss in multiple cell types including neurons and glia (Fraser et al. 2004, 2008), depending on the expression pattern of Cre recombinase used. In addition, a migration defect was observed in Pten-deficient cerebellar neurons. At the molecular level, hyperphosphorylation of Akt and S6, a substrate of S6K, was detected predominantly in Pten-deficient neurons (Kwon et al. 2001, 2003; Fraser et al. 2004), indicating activation of downstream effectors regulated by Pdk1.

To determine the role of Pdk1 in mature brains, and the contribution of Pdk1 to abnormalities in Pten-deficient brains, we generated mice with brain-specific inactivation of Pten, Pdk1, or combined deletion of both. Here, we report that Pdk1 deficiency caused microcephaly, and combined deletion of Pdk1 and Pten rescued the neuronal hypertrophy, but not the migration defect of cerebellar neurons in Pten-deficient brains. Pdk1 loss caused strong feedback up-regulation of Akt phosphorylation at Ser 473 in glia, but not in neurons. Our in vivo results show that Pdk1 is not required for all abnormalities associated with Pten deficiency in the brain, and demonstrate cell type specific differential regulation of the ubiquitously expressed PI3K pathway.

\section{Results and Discussion}

\section{Pdk1 deficiency caused microcephaly and rescued} macrocephaly in Pten-deficient brains

To assess the contribution of Pdk1 to Pten-deficient brain hypertrophy in vivo, we used a GFAP-cre transgenic line directing Cre-mediated deletion throughout the brain in both neurons and glia, with nearly complete activity in the hippocampus (hereafter referred to as $h p$-cre) (Bajenaru et al. 2002; Fraser et al. 2004). hp-cre mice were bred to 
Pten;Pdk1-floxed mice to generate the four genotypes analyzed in this study: Pten ${ }^{\operatorname{lox} P / \operatorname{lox} P} ; P d k 1^{\text {loxP/+ }} ;$ cre (Pten cKO), Pten ${ }^{\text {loxP/+ }}$;Pdk $1^{\text {loxP/loxP }}$; cre (Pdk1 cKO), Pten ${ }^{\text {loxP/loxP }}$; Pdk1 $1^{\text {loxP/loxP }}$; cre (Pten;Pdk1 dKO), and No-cre mice (control). Pdk1 cKO mice had a significantly decreased brain size relative to controls (Fig. 1). Conditional deletion of Pten caused pronounced macrocephaly, as reported previously (Kwon et al. 2001; Fraser et al. 2004). CT scans to measure brain volume showed that combined deletion of Pten and Pdk1 fully rescued the Pten-deficient macrocephaly, with Pten;Pdk1 $d K O$ brains showing a similar reduction in size as $P d k 1 \mathrm{cKO}$ brains compared with controls (Fig. 1; Supplemental Fig. S1). Additionally, severe ataxia and seizures observed in Pten cKO mice were never observed in age-matched Pten;Pdk1 dKO littermates. Furthermore, astrogliosis and microgliosis associated with the disorganization and enlargement of the hippocampus in Pten cKO mice (Fraser et al. 2004) were rescued in Pten;Pdk1 $d K O$ brains (Supplemental Fig. S2A; data not shown).

\section{Pdk1 deficiency rescued cell-autonomous hypertrophy of Pten-deficient neuronal nuclei and somata}

We verified deletion of Pten in hippocampus by Pten immunohistochemistry (IHC) (Supplemental Fig. S2A,B). Pten-deficient neurons also displayed elevated phosphorylation of S6 (p-S6), consistent with enhanced mTorc1 signaling leading to downstream activation of S6K (Fig. $2 \mathrm{~A})$. Full activation of S6K also requires phosphorylation by Pdk1 (Collins et al. 2003). IHC showed that p-S6 was absent in Pten;Pdk1 $d K O$ or Pdk1 cKO hippocampi, consistent with efficient deletion of Pdk1 (Fig. 2A). We used this indirect assessment because Pdk1 antibodies were not reliable for IHC.

To determine the contribution of Pdk1 to the cellautonomous hypertrophy of Pten-deficient neurons, we measured the size of Pten-positive and Pten-negative granule neurons from the dentate gyrus within the hippocampal region from mice with single or combined deletion of Pten and Pdk1, and controls (Fig. 2B; Supplemental Fig. S2B). We evaluated nuclear diameter because

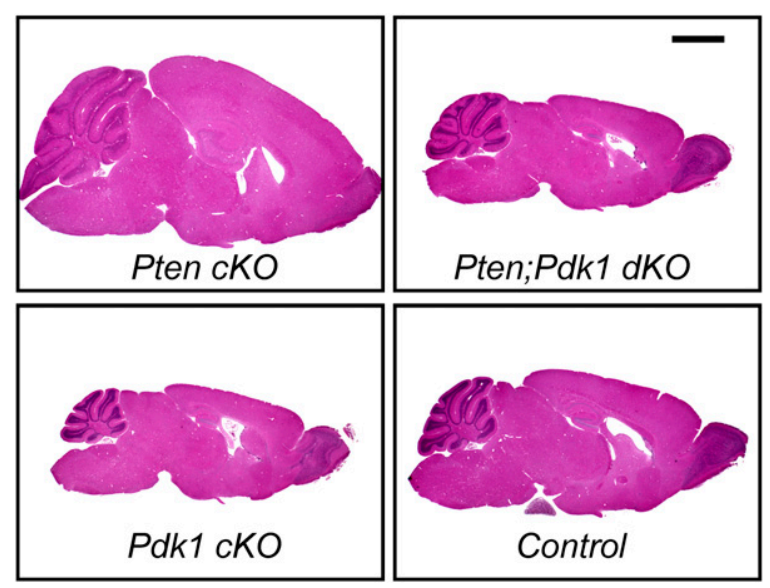

Figure 1. Pdk1 is indispensible for brain size regulation. Sagittal H\&E-stained sections from mice with the indicated genotypes, in which $h p$-cre drives conditional deletion. The dramatic hypertrophy of Pten $c K O$ was rescued in Pten;Pdk1 dKO brains, which show a similar size reduction to $P d k 1$-deficient brains when compared with control. Bar, $2 \mathrm{~mm}$. Mice were 10 wk old.

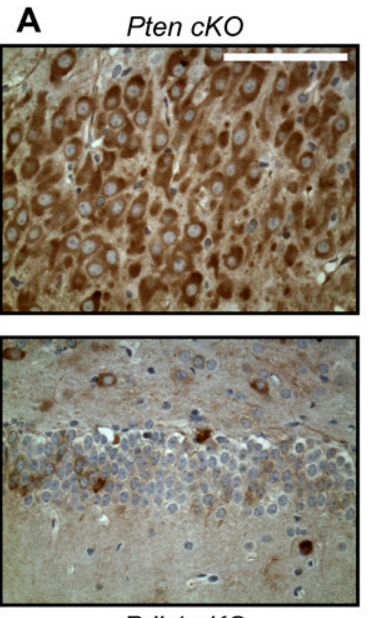

Pdk1 cKO
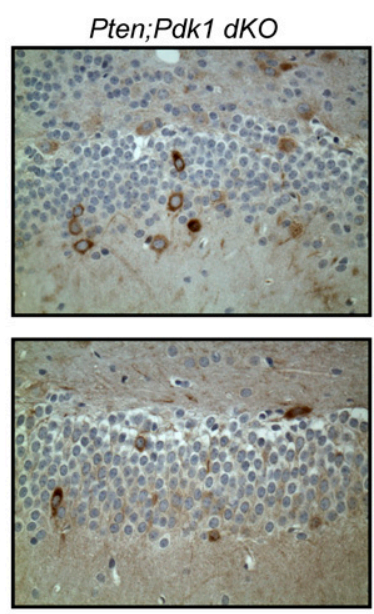

Control

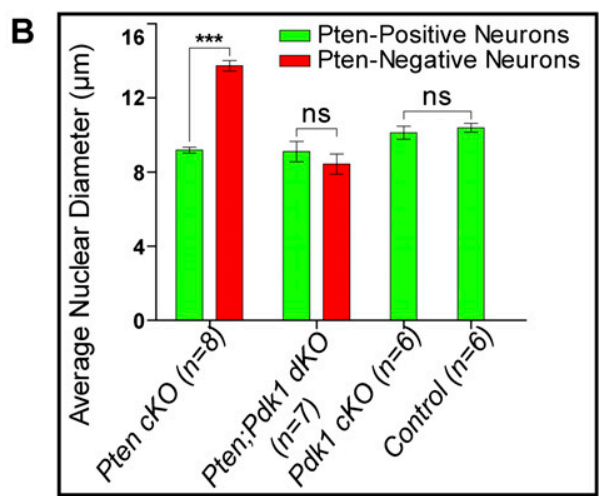

Figure 2. Pdk1 is required for cell-autonomous hypertrophy of Ptendeficient neurons. (A) IHC for p-S6 (Ser 235/236) in the dentate gyrus from the indicated genotypes combined with $h p$-cre. Elevated levels of p-S6 in Pten cKO neurons were abrogated in Pten;Pdk1 dKO, consistent with deletion of $P d k 1$. Bar, $100 \mu \mathrm{m}$. (B) The nuclear diameter of granule neurons was measured using Pten IHC to distinguish between Pten-positive and Pten-negative neurons. The mean \pm SEM of 50 measured nuclei per brain section from six to eight mice is shown. $P$ values $\left(\left[^{\star \star \star}\right] P<0.0001\right.$; [ns] not significant, $\left.P>0.05\right)$ were determined by a Student's unpaired $t$-test. Mice were $10 \mathrm{wk}$ old.

the neuronal soma boundary is not consistently visible in all cells and we showed previously that nuclei and somata were coordinately enlarged in Pten-deficient neurons (Kwon et al. 2001, 2003; Fraser et al. 2004, 2008). The enlarged nuclear diameter of Pten-deficient neurons was fully rescued to normal size by the concomitant deletion of $P d k 1$, demonstrating a requirement for Pdk1 in the cell-autonomous hypertrophy of Pten-deficient neurons. Pdk1 deletion did not cause a significant decrease in the nuclear size of dentate gyrus granule neurons, perhaps due to the contribution of some remaining Pdk1-positive neurons included in the measurements, as we did not have an IHC assay to allow exclusion of Pdk1-expressing neurons.

\section{Pdk1 deficiency did not rescue the cerebellar neuron migration defect in Pten;Pdk1 dKO mice}

We also evaluated the cerebellum to determine the contribution of Pdk1 to hypertrophy and migration defects in cerebellar granule neurons that we identified previously in Pten cKO mice. However, a significant reduction in the 
number of Pten-deficient neurons present in Pten;Pdk1 $d K O$ cerebella compared with Pten $c K O$ was reproducibly observed (Supplemental Fig. S3A). This suggests that cells with combined deletion of Pten and Pdk1 had severely compromised survival, consistent with the requirement for PI3K activation in granule cell progenitor survival demonstrated previously (Dudek et al. 1997; Kenney et al. 2004; Zhao et al. 2006). Therefore, we employed an alternative Gfap-cre transgenic line with later onset of Cre activity in the postnatal cerebellar granule cells (Kwon et al. 2001). For simplicity of discussion, this line is referred to as $c b$-cre hereafter. Similar numbers of Pten-deficient cerebellar granule neurons were observed in the Pten cKO and Pten;Pdk $1 d K O$ mice with $c b$-cre, likely because the later onset of Cre activity, around postnatal day 14, bypassed the developmental stage at which Pdk1 was required for survival (Supplemental Fig. S3B; Kwon et al. 2001). This allowed a controlled comparison of Pten-deficient cerebellar granule neurons in the absence and presence of Pdk1. Consistent with the size rescue observed in the hippocampus with $h p$-cre;Pten;Pdk1 dKO mice, the overall size of the $c b$ cre;Pten $c K O$ cerebellum and the hypertrophy of individual Pten-deficient cerebellar granule neurons were completely rescued in $c b$-cre;Pten;Pdk1 $d K O$ mice (Supplemental Fig. S4).

Strikingly, Pdk1 deletion failed to rescue migration defects of Pten-deficient cerebellar granule neurons. During cerebellar development, granule cell progenitors proliferate in the external germinal layer (EGL) at the surface of the cerebellum, then exit the cell cycle and migrate to their final position in the internal granule layer (IGL), where they fully differentiate (Behesti and Marino 2008). Ectopically positioned granule neurons are easily visualized by expression of $\mathrm{GABA}_{\mathrm{A}} \mathrm{R} \alpha 6$, which is normally restricted to mature granule neurons of the IGL. As expected, cerebella from $c b$-cre;Pten $c K O$ mice contained ectopic $\mathrm{GABA}_{\mathrm{A}} \mathrm{R} \alpha 6$-positive granule neurons at the pial surface and in the molecular layer (Fig. 3; Supplemental Fig. S5). These neurons were hypertrophic and expressed elevated levels of p-S6, as observed in other Pten-deficient neurons (Fig. 3). Surprisingly, concomitant deletion of $P d k 1$ did not rescue this migration defect. Ectopic $\mathrm{GABA}_{\mathrm{A}}$ R $\alpha 6$-expressing granule neurons were consistently observed in Pten;Pdk1 $d K O$ cerebella, but not in control cerebella (Fig. 3; Supplemental Fig. S5). Pdk1 was clearly deleted in ectopic granule neurons of Pten;Pdk1 dKO cerebella, as these cells did not show the hypertrophy or elevated p-S6 associated with Pten-null neurons (Fig. 3).

While there are a number of examples of AKT activation influencing migration (Kolsch et al. 2008), the failure of Pdk1 loss to rescue the migration defect of Ptendeficient cerebellar granule neurons indicates that at least a component of this phenotype is AKT-independent. PTEN may also influence cell migration through alternative mechanisms. For example, elevated PIP3 can regulate cytoskeletal rearrangement and cell migration and motility through an AKT-independent pathway involving small GTPases, such as RAC1/CDC42 (Kolsch et al. 2008). Alternatively, in vitro studies have implicated the protein phosphatase activity of PTEN in adhesion and migration, independent of its effects on the PI3K pathway (Tamura et al. 1998; Raftopoulou et al. 2004). Mutant mouse models that selectively target the lipid or protein phosphatase activity of Pten will help to further
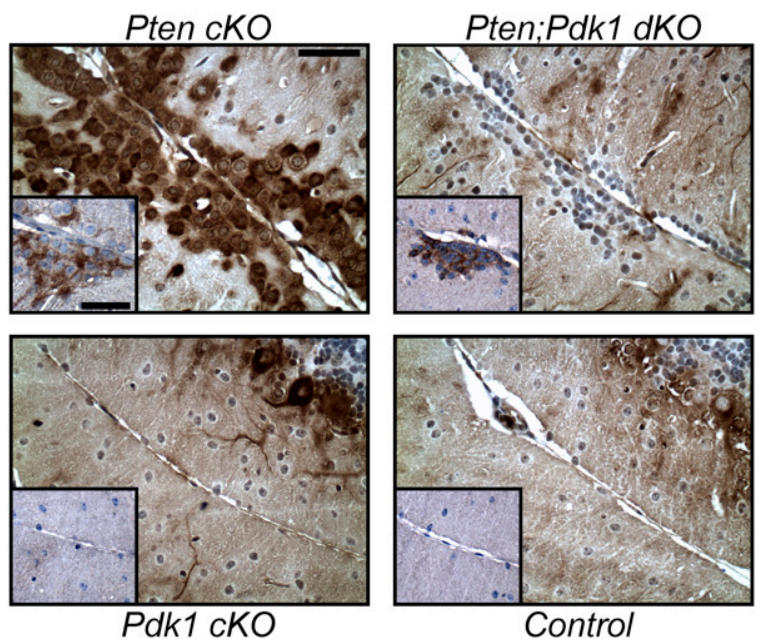

Figure 3. Pdk1 deficiency did not rescue the migration defect in Pten cKO cerebellar granule neurons. IHC for p-S6 (Ser 235/236) on cerebella from the indicated genotypes combined with cb-cre. Pten $c K O$ cerebella contained ectopic neurons that were hypertrophic and contained elevated p-S6. Ectopic granule neurons persist in Pten;Pdk1 $d K O$ cerebella, but they do not show hypertrophy or elevated p-S6, consistent with deletion of Pdk1. Insets show IHC for $\mathrm{GABA}_{\mathrm{A}} \mathrm{R} \alpha 6$ demonstrating that ectopic cells are cerebellar granule neurons. Bar, $50 \mu \mathrm{m}$. Mice were $8 \mathrm{wk}$ old.

resolve the mechanistic basis of migration defects in Pten-deficient neurons.

\section{Pdk1 deletion blocked phosphorylation of downstream effectors}

To verify deletion of Pdk1 and the concomitant loss of phosphorylation of downstream components in the PI3K signaling cascade, we performed Western blot analysis on cerebellar and hippocampal protein extracts in Pdk1 cKO and Pten;Pdk1 $d K O$ brains. Cerebellar protein extracts from the $c b$-cre line showed substantial loss of Pten, Pdk1, or both as predicted by their corresponding genotype (Supplemental Fig. S6A). Akt is fully activated when phosphorylated by Pdk1 on Thr 308 (T308) and by a second kinase, most commonly the mTorc 2 complex, on Ser 473 (S473) (Mora et al. 2004; Sarbassov et al. 2005). High p-Akt (S473 and T308) levels were present in Pten $c K O$ extracts, as expected for activated PI3K signaling. In Pten;Pdk1 dKO cerebella, although p-Akt S473 was still elevated, phosphorylation at T308 was abolished by the deletion of $P d k 1$, which would prevent full activation of Akt. As expected, the enhanced levels of phosphorylation of the Akt substrate Gsk3 $\beta$ observed in Pten cKO cerebellum were restored to levels similar to controls in Pten;Pdk1 $d K O$ cerebella. In addition, conditional deletion of Pdk1, alone or in combination with Pten, substantially decreased levels of p-S6. Similar loss of p-Akt T308 and decreased phosphorylation of S6, as well as decreased phosphorylation of GSK3 $\beta$ and an additional Akt substrate, Fkhr, were associated with Pdk1 deficiency in hippocampal protein extracts from the $h p$-cre line (Fig. 4). Taken together, these data demonstrate that $P d k 1$ was effectively codeleted along with Pten in the cerebellum and hippocampus using cb-cre and hp-cre, respectively. 


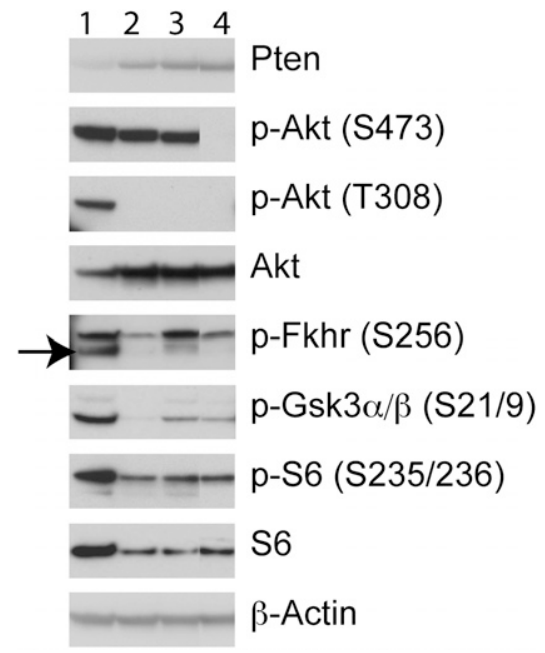

Figure 4. Pdk1 deficiency blocked phosphorylation of downstream effectors of the PI3K pathway and induced up-regulation of p-Akt S473. Western blot analysis of protein lysates from hippocampi of hp-cre mice. (Lane 1) Pten cKO. (Lane 2) Pten;Pdk1 dKO. (Lane 3) Pdk1 cKO. (Lane 4) Control. Pten cKO mice showed increased phosphorylation of Akt at S473 and T308, and increased phosphorylation of downstream substrates GSK3 $\beta$, S6, and Fkhr. (Lane 2) This enhanced phosphorylation of Akt T308 and substrates was blocked by Pdk1 deletion. (Lane 3) Note the high levels of p-Akt S473 in Pdk1 cKO. $\beta$-Actin is shown as a loading control. The arrow denotes a specific band. Mice were 10 wk old.

\section{Cell type specific feedback regulation of p-Akt S473 in Pdk1-deficient brains}

Western blot analysis of Pdk1-deficient brain regions showed complete loss of Akt phosphorylation in $c b$ cre;Pdk1-deficient cerebella (Supplemental Fig. S6A), in which Cre activity was restricted to neurons (Kwon et al. 2001). In contrast, in hippocampal extracts in which Cre activity was present in neurons and glia (Fraser et al. 2004), Pdk1 deletion alone abrogated phosphorylation of its substrate, Akt T308, but unexpectedly induced phosphorylation of Akt S473 to levels comparable with those induced by deregulated PI3K signaling in Pten-deficient hippocampi (Fig. 4). The up-regulation of p-Akt S473 was blocked by the dual PI3K/mTor inhibitor BEZ235 (Supplemental Fig. S6B; Maira et al. 2008).

To investigate the selective up-regulation of p-Akt S473 in $h p$-cre mice, we used IHC. As expected in the cb-cre line, Pten $c K O$ granule neurons displayed elevated levels of p-Akt S473 in the IGL of the cerebellum. Pten-deficient regions of Pten;Pdk1 $d K O$ cerebella had similar elevated p-Akt S473 levels to Pten cKO, although the density of staining was higher due to the size rescue of cerebellar granule neurons. In contrast, Pdk1 cKO cerebella were similar to controls with no detectable p-Akt S473 signal in cerebellar granule neurons (Supplemental Fig. S6C), indicating that Pdk1 deletion does not alter the phosphorylation status of Akt S473 in mature cerebellar granule neurons. Interestingly, using the hp-cre line, which targets both neurons and glia, Pdk1 deficiency led to a surprising p-Akt pattern (Fig. 5). As reported previously, Pten cKO brains had elevated levels of p-Akt S473 predominantly in neurons, including the hippocampus and cortex, and occasionally in a few reactive hippocampal astrocytes (Fraser et al. 2004). Unexpectedly, in addition to the neuronal populations with elevated p-Akt S473 observed in Pten cKO mice, Pten;Pdk1 dKO brains also showed high levels of p-Akt S473 in cells with stellate morphology characteristic of glia (Fig. 5). The single deletion of Pdk1, without loss of Pten, caused elevated levels of p-Akt S473 only in cells with glial morphology in both the hippocampus and cortex, indicating a compensatory or feedback up-regulation of pAkt S473. To confirm the identity of these cells, we took advantage of the lacZ marker included in the GFAP-cre transgene. In adult brains, this transgene was shown to be expressed selectively in astrocytes (Bajenaru et al. 2002), while the broader Cre activity induced by the transgene is a reflection of Cre-mediated deletion earlier in development (Fraser et al. 2004). We double-stained brain cryosections using X-Gal histochemistry and IHC for p-Akt S473, and found a complete overlap between p-Akt and X-Galpositive cells in Pten;Pdk $1 d K O$ and Pdk1 cKO cerebral cortices. Further double-labeling showed elevated p-Akt S473 was restricted to astrocytes and Olig2-expressing cells of the oligodendroglial lineage, and was not found in neurons or microglia (Supplemental Fig. S7; data not shown). No overlap was seen between X-Gal and p-Aktpositive cells in Pten cKO brains (Fig. 5, right panels), highlighting the distinct cell type differences in response to disruption of different effectors in the PI3K pathway.

Feedback inhibition in PI3K signaling has been demonstrated, but differential regulation of this mechanism in normal cells has not been clearly shown. S6K can phosphorylate and inhibit IRS1, creating a negative feedback loop that limits insulin and IGF receptor signaling through the PI3K pathway (Um et al. 2004). Release of this feedback inhibitory pathway occurs in some, but not all, cancer cells in response to mTOR inhibition. This results in higher levels of AKT activation, and likely contributes to the failure of mTOR inhibitors in cancer

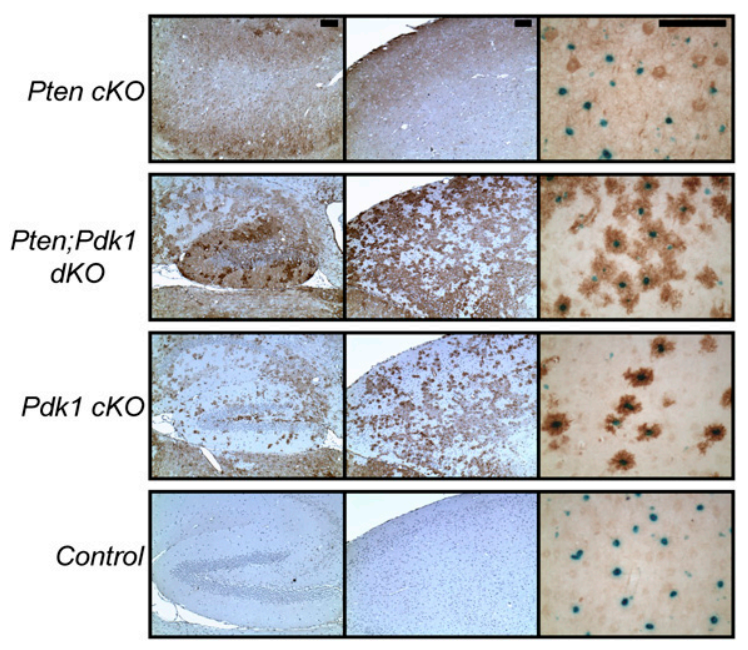

Figure 5. Pdk1 deficiency caused elevation of p-Akt S473 selectively in glia. IHC with p-Akt S473 antibody on the hippocampus (left) and cortex (center). (Right) X-Gal histochemistry followed by p-Akt IHC revealed $\beta$-galactosidase-positive glia. The p-Akt-positive neurons in Pten cKO do not overlap with X-gal, while high levels of p-Akt are present in X-gal-positive glia within Pten;Pdk1 $d K O$ and Pdk1 cKO cerebral cortices compared with control. Bars: left and center panels, $400 \mu \mathrm{m}$; right panel, $100 \mu \mathrm{m}$. Mice were $10 \mathrm{wk}$ (left and center panels), and 2 wk old (right panel). 
therapy. It remains unclear why cancer cells are heterogeneous in this response to mTOR inhibition $/ \mathrm{O}^{\prime}$ Reilly et al. 2006; Cloughesy et al. 2008). Deletion of Pdk1 prevents full activation of S6k, and would therefore similarly relieve feedback inhibition and cause enhanced phosphorylation of p-Akt S473. Indeed, elevated phosphorylation of p-Akt S473 following Pdk1 deletion has been reported in mouse embryonic stem cells, heart, and liver (Williams et al. 2000; Mora et al. 2003, 2005). However, p-Akt status was assessed by Western blot in these experiments, precluding interpretation of the differences in pathway regulation between cell types within a tissue under physiological conditions.

Our data show a striking cell type difference in pathway regulation. Deletion of the negative regulator Pten induced increased p-Akt S473 in neurons, but not glia. In contrast, deletion of the positive regulator Pdk1 caused increased p-Akt S473 in glia, but not neurons. This is consistent with a strong feedback in glia, which acts to limit PI3K signaling, even in the absence of Pten. The reason for this difference in regulation may reflect the biological properties and roles of these cell types. Except in response to specific mutations, mature neurons do not proliferate or regenerate, suggesting no need for a tight control of PI3K signaling through feedback regulation in these cells. In contrast, glia can re-enter the cell cycle following different cues. This response should be limited to minimize pathological conditions such as glial scars and the possibility of oncogenic transformation to generate gliomas (Yang and Herrup 2007). Glia, therefore, may require tighter control mechanisms on PI3K signaling, including feedback regulation.

In summary, our study shows that Pdk 1 is a critical player in the Pten signaling pathway. Our results demonstrate clearly that Pdk1 loss rescues Pten-deficient brain hypertrophy in agreement with genetic studies showing epistasis between Pten and Pdk1 in flies and mice (Rintelen et al. 2001; Bayascas et al. 2005). However, Pdk1 loss did not rescue Pten-null neuronal migration defects. This may reflect AKT-independent regulation of migration through PI3K signaling, or may be due to PIP3independent functions of PTEN in neuronal migration. Surprisingly, analysis of Akt phosphorylation revealed striking cell type differences in feedback up-regulation of p-Akt S473 in Pdk1-deficient glia compared with neurons in the mature brain. In vitro studies have elegantly shown the mechanistic connections among key effectors in the PI3K signaling pathway. Our in vivo analysis demonstrates that under physiological conditions, this ubiquitously expressed pathway may show functionally important differences in regulation between cell types that are important in normal development and disease.

\section{Materials and methods}

All experimental procedures were as described previously (Chalhoub et al. 2006) with the following modifications or additions: Pten antibody (Cell Signaling, \#9559) was used at a $1 / 100$ dilution for IHC, and 1/1000 dilution for immunoblot; Pdk1 antibody (Cell Signaling, \#3062) was used at a 1/1000 dilution for immunoblot; p-Akt (T308) antibody (Cell Signaling, \#9275) was used at a 1/1000 dilution for immunoblot; $\mathrm{GABA}_{\mathrm{A}} \mathrm{R} \alpha 6$ antibody (Santa Cruz Biotechnologies, \#sc-7359) was used at 1/500 dilution for IHC following antigen retrieval. IHC was counterstained with hematoxylin.

For the X-Gal and p-Akt (S473) double stain, we perfused mice with $2 \%$ PFA to fix, removed brains, and post-fixed overnight in $2 \%$ PFA. After $24 \mathrm{~h}$ of equilibration in $25 \%$ sucrose/PBS, OCT-embedded brains were cryo- sectioned. We performed X-Gal histochemistry as described (Chow et al. 2008), followed by 5 min of fixation in $4 \%$ PFA, then proceeded with p-Akt IHC as described previously (Chalhoub et al. 2006) without counterstain.

\section{Acknowledgments}

We are grateful to Dr. Dario Alessi for $P d k 1^{+/ l o x P}$ mice, Dr. David Gutmann for GFAP-cre mice, and Dr. Tak Mak for Pten ${ }^{+/ l o x P}$ mice. We thank Novartis for providing BEZ-235. We thank Dr. Christopher Calabrese for CT scans and volumetric measurements. We thank Jovan Mitchell for expert genotyping of mice. We thank Peter McKinnon, Stanislav Zakharenko, and members of the Baker laboratory for useful discussions. This work was supported by NIH grants CA135554 and CA096832 to S.J.B., a post-doctoral fellowship from the Canadian Institutes of Health Research to N.C., and the American Lebanese and Syrian Associated Charities.

\section{References}

Backman SA, Stambolic V, Suzuki A, Haight J, Elia A, Pretorius J, Tsao MS, Shannon P, Bolon B, Ivy GO, et al. 2001. Deletion of Pten in mouse brain causes seizures, ataxia and defects in soma size resembling Lhermitte-Duclos disease. Nat Genet 29: 396-403.

Bajenaru ML, Zhu Y, Hedrick NM, Donahoe J, Parada LF, Gutmann DH. 2002. Astrocyte-specific inactivation of the neurofibromatosis 1 gene (NF1) is insufficient for astrocytoma formation. Mol Cell Biol 22: 5100-5113.

Bayascas JR, Leslie NR, Parsons R, Fleming S, Alessi DR. 2005. Hypomorphic mutation of PDK1 suppresses tumorigenesis in $\mathrm{PTEN}^{+/-}$ mice. Curr Biol 15: 1839-1846.

Behesti H, Marino S. 2008. Cerebellar granule cells: Insights into proliferation, differentiation, and role in medulloblastoma pathogenesis. Int I Biochem Cell Biol 41: 435-445.

Chalhoub N, Baker SJ. 2009. PTEN and the PI3-kinase pathway in cancer. Annu Rev Pathol 4: 127-150.

Chalhoub N, Kozma SC, Baker SJ. 2006. S6k1 is not required for Ptendeficient neuronal hypertrophy. Brain Res 1100: 32-41.

Chow LM, Zhang J, Baker SJ. 2008. Inducible Cre recombinase activity in mouse mature astrocytes and adult neural precursor cells. Transgenic Res 17: 919-928.

Cloughesy TF, Yoshimoto K, Nghiemphu P, Brown K, Dang J, Zhu S, Hsueh T, Chen Y, Wang W, Youngkin D, et al. 2008. Antitumor activity of rapamycin in a Phase I trial for patients with recurrent PTEN-deficient glioblastoma. PLoS Med 5: e8. doi: 10.1371/journal. pmed.0050008.

Collins BJ, Deak M, Arthur JS, Armit LJ, Alessi DR. 2003. In vivo role of the PIF-binding docking site of PDK1 defined by knock-in mutation. EMBO J 22: 4202-4211.

Di Cristofano A, Pesce B, Cordon-Cardo C, Pandolfi PP. 1998. Pten is essential for embryonic development and tumour suppression. Nat Genet 19: 348-355.

Dudek H, Datta SR, Franke TF, Birnbaum MJ, Yao R, Cooper GM, Segal RA, Kaplan DR, Greenberg ME. 1997. Regulation of neuronal survival by the serine-threonine protein kinase Akt. Science 275: 661-665.

Engelman JA, Luo J, Cantley LC. 2006. The evolution of phosphatidylinositol 3-kinases as regulators of growth and metabolism. Nat ReV Genet 7: 606-619.

Fraser MM, Zhu X, Kwon CH, Uhlmann EJ, Gutmann DH, Baker SJ. 2004. Pten loss causes hypertrophy and increased proliferation of astrocytes in vivo. Cancer Res 64: 7773-7779.

Fraser MM, Bayazitov IT, Zakharenko SS, Baker SJ. 2008. Phosphatase and tensin homolog, deleted on chromosome 10 deficiency in brain causes defects in synaptic structure, transmission and plasticity, and myelination abnormalities. Neuroscience 151: 476-488.

Groszer M, Erickson R, Scripture-Adams DD, Lesche R, Trumpp A, Zack JA, Kornblum HI, Liu X, Wu H. 2001. Negative regulation of neural stem/progenitor cell proliferation by the Pten tumor suppressor gene in vivo. Science 294: 2186-2189.

Haga S, Ozaki M, Inoue H, Okamoto Y, Ogawa W, Takeda K, Akira S, Todo S. 2008. The survival pathways phosphatidylinositol-3 kinase (PI3-K)/phosphoinositide-dependent protein kinase 1 (PDK1)/Akt modulate liver regeneration through hepatocyte size rather than proliferation. Hepatology 49: 204-214. 
Hashimoto N, Kido Y, Uchida T, Asahara S, Shigeyama Y, Matsuda T, Takeda A, Tsuchihashi D, Nishizawa A, Ogawa W, et al. 2006. Ablation of PDK1 in pancreatic $\beta$ cells induces diabetes as a result of loss of $\beta$ cell mass. Nat Genet 38: 589-593.

Kenney AM, Widlund HR, Rowitch DH. 2004. Hedgehog and PI-3 kinase signaling converge on Nmycl to promote cell cycle progression in cerebellar neuronal precursors. Development 131: 217-228.

Kolsch V, Charest PG, Firtel RA. 2008. The regulation of cell motility and chemotaxis by phospholipid signaling. I Cell Sci 121: 551-559.

Kwon CH, Zhu X, Zhang J, Knoop LL, Tharp R, Smeyne RJ, Eberhart CG, Burger PC, Baker SJ. 2001. Pten regulates neuronal soma size: A mouse model of Lhermitte-Duclos disease. Nat Genet 29: 404-411.

Kwon CH, Zhu X, Zhang J, Baker SJ. 2003. mTor is required for hypertrophy of Pten-deficient neuronal soma in vivo. Proc Natl Acad Sci 100: 12923-12928.

Lawlor MA, Mora A, Ashby PR, Williams MR, Murray-Tait V, Malone L, Prescott AR, Lucocq JM, Alessi DR. 2002. Essential role of PDK1 in regulating cell size and development in mice. ЕMBO I 21: 3728-3738.

Li L, Liu F, Salmonsen RA, Turner TK, Litofsky NS, Di Cristofano A, Pandolfi PP, Jones SN, Recht LD, Ross AH. 2002. PTEN in neural precursor cells: Regulation of migration, apoptosis, and proliferation. Mol Cell Neurosci 20: 21-29.

Maira SM, Stauffer F, Brueggen J, Furet P, Schnell C, Fritsch C, Brachmann S, Chene P, De Pover A, Schoemaker K, et al. 2008. Identification and characterization of NVP-BEZ235, a new orally available dual phosphatidylinositol 3-kinase/mammalian target of rapamycin inhibitor with potent in vivo antitumor activity. Mol Cancer Ther 7: 1851-1863.

Marino S, Krimpenfort P, Leung C, van der Korput HA, Trapman I, Camenisch I, Berns A, Brandner S. 2002. PTEN is essential for cell migration but not for fate determination and tumourigenesis in the cerebellum. Development 129: 3513-3522.

Mora A, Davies AM, Bertrand L, Sharif I, Budas GR, Jovanovic S, Mouton V, Kahn CR, Lucocq JM, Gray GA, et al. 2003. Deficiency of PDK1 in cardiac muscle results in heart failure and increased sensitivity to hypoxia. $E M B O$ I 22: 4666-4676.

Mora A, Komander D, van Aalten DM, Alessi DR. 2004. PDK1, the master regulator of AGC kinase signal transduction. Semin Cell Dev Biol 15: 161-170.

Mora A, Lipina C, Tronche F, Sutherland C, Alessi DR. 2005. Deficiency of PDK1 in liver results in glucose intolerance, impairment of insulinregulated gene expression and liver failure. Biochem J 385: 639-648.

O'Reilly KE, Rojo F, She QB, Solit D, Mills GB, Smith D, Lane H, Hofmann F, Hicklin DJ, Ludwig DL, et al. 2006. mTOR inhibition induces upstream receptor tyrosine kinase signaling and activates Akt. Cancer Res 66: 1500-1508.

Podsypanina K, Ellenson LH, Nemes A, Gu J, Tamura M, Yamada KM, Cordon-Cardo C, Catoretti G, Fisher PE, Parsons R. 1999. Mutation of Pten/Mmacl in mice causes neoplasia in multiple organ systems. Proc Natl Acad Sci 96: 1563-1568.

Raftopoulou M, Etienne-Manneville S, Self A, Nicholls S, Hall A. 2004. Regulation of cell migration by the $\mathrm{C} 2$ domain of the tumor suppressor PTEN. Science 303: 1179-1181.

Rintelen F, Stocker H, Thomas G, Hafen E. 2001. PDK1 regulates growth through Akt and S6K in Drosophila. Proc Natl Acad Sci 98: 1502015025 .

Sarbassov DD, Guertin DA, Ali SM, Sabatini DM. 2005. Phosphorylation and regulation of Akt/PKB by the rictor-mTOR complex. Science 307: 1098-1101.

Suzuki A, de la Pompa JL, Stambolic V, Elia AJ, Sasaki T, del Barco Barrantes I, Ho A, Wakeham A, Itie A, Khoo W, et al. 1998. High cancer susceptibility and embryonic lethality associated with mutation of the PTEN tumor suppressor gene in mice. Curr Biol 8: 11691178.

Tamura M, Gu J, Matsumoto K, Aota S, Parsons R, Yamada KM. 1998. Inhibition of cell migration, spreading, and focal adhesions by tumor suppressor PTEN. Science 280: 1614-1617.

Um SH, Frigerio F, Watanabe M, Picard F, Joaquin M, Sticker M, Fumagalli S, Allegrini PR, Kozma SC, Auwerx I, et al. 2004. Absence of S6K1 protects against age- and diet-induced obesity while enhancing insulin sensitivity. Nature 431: 200-205.

Williams MR, Arthur JS, Balendran A, van der Kaay J, Poli V, Cohen P, Alessi DR. 2000. The role of 3-phosphoinositide-dependent protein kinase 1 in activating AGC kinases defined in embryonic stem cells. Curr Biol 10: 439-448.

Yang Y, Herrup K. 2007. Cell division in the CNS: Protective response or lethal event in post-mitotic neurons? Biochim Biophys Acta 1772: 457-466.

Yue Q, Groszer M, Gil JS, Berk AJ, Messing A, Wu H, Liu X. 2005. PTEN deletion in Bergmann glia leads to premature differentiation and affects laminar organization. Development 132: 3281-3291.

Zhao H, Sapolsky RM, Steinberg GK. 2006. Phosphoinositide-3-kinase/ akt survival signal pathways are implicated in neuronal survival after stroke. Mol Neurobiol 34: 249-270. 


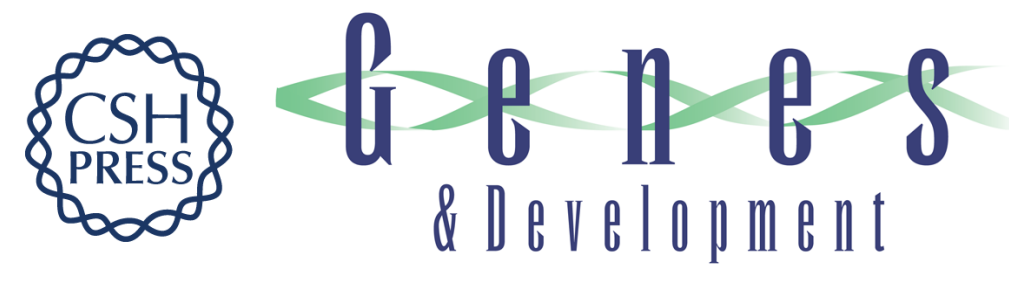

\section{Cell type specificity of PI3K signaling in Pdk1- and Pten-deficient brains}

Nader Chalhoub, Guo Zhu, Xiaoyan Zhu, et al.

Genes Dev. 2009, 23:

Access the most recent version at doi:10.1101/gad.1799609

\section{Supplemental http://genesdev.cshlp.org/content/suppl/2009/07/01/23.14.1619.DC1 \\ Material}

Related Content Striking the balance between PTEN and PDK1: it all depends on the cell context Akio Iwanami, Timothy F. Cloughesy and Paul S. Mischel

Genes Dev. August , 2009 23: 1699-1704

References This article cites 40 articles, 18 of which can be accessed free at:

http://genesdev.cshlp.org/content/23/14/1619.full.html\#ref-list-1

Articles cited in:

http://genesdev.cshlp.org/content/23/14/1619.full.html\#related-urls

\section{License}

Email Alerting

Service

Receive free email alerts when new articles cite this article - sign up in the box at the top right corner of the article or click here.

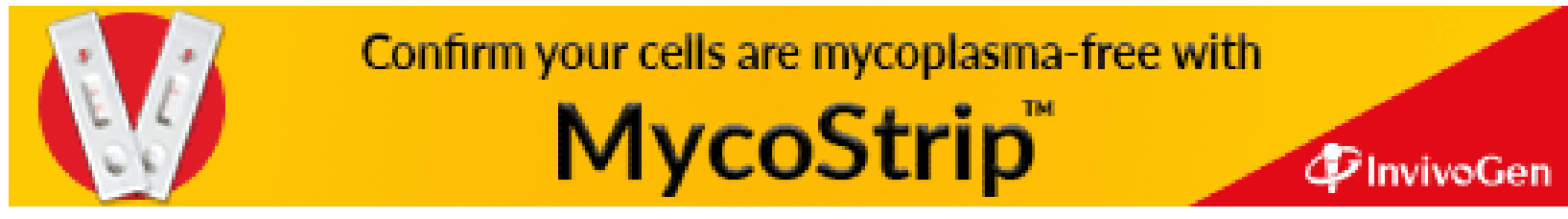

